Predischarge occupational therapy home assessments are routinely performed with older adults in Europe, Australia and North America. Their primary aim is to facilitate a timely and safe discharge from hospital. However, there is a lack of published research on this topic, especially studies exploring occupational therapists' perceptions of home assessments. The paper aims to redress this by describing occupational therapists' perceptions of predischarge occupational therapy home assessments with older adults in acute care.

All occupational therapists who undertook home assessments in an acute care hospital with older adults during the duration of the study period were invited to complete a reflective diary. In total, 15 reflective diaries were completed by six therapists. The data were analysed using thematic content analysis.

The findings suggest that home assessments were carried out because of mobility or environmental concerns. Satisfaction and dissatisfaction with the outcome of the home assessment were related to the incidents that occurred during the assessment. Some of the occupational therapists' anxieties were related to the older adults' level of functioning or ill health, and the older adults' own concerns did have an impact upon the therapists' expectations of the home assessment process.

\title{
Occupational Therapists' Perceptions of Predischarge Home Assessments with Older Adults in Acute Care
}

\author{
Anita Atwal, ${ }^{1}$ Anne McIntyre, ${ }^{1}$ Christine Craik ${ }^{1}$ and Jacki Hunt ${ }^{2}$
}

\section{Introduction}

The importance of discharge planning has increased since the publication of the National Health Service (NHS) Plan (Department of Health 2000), where a clear distinction is made between acute and intermediate care. This document highlights the importance of freeing acute beds and looking at alternative and creative ways of rehabilitating and discharging patients.

Occupational therapy predischarge home visits are regarded as a means to facilitate the timely, safe and successful discharge of patients from hospital and to add to the total picture that therapists have of patients in the

\footnotetext{
${ }^{1}$ Brunel University, Uxbridge, Middlesex.

${ }^{2}$ Hounslow Primary Care Trust, West Middlesex University Hospital, Isleworth, Middlesex.
}

Corresponding author: Dr Anita Atwal, Lecturer in Occupational Therapy, School of Health Science and Social Care, Mary Seacole Building, Brunel University, Uxbridge, Middlesex UB8 3PH. Email: anita.atwal@brunel.ac.uk

Submitted: 26 April 2007

Accepted: 31 January 2008.

Key words: Home assessment, predischarge, occupational therapy, acute care. Reference: Atwal A, McIntyre A, Craik C, Hunt J (2008) Occupational therapists' perceptions of predischarge home assessments with older adults in acute care. British Journal of Occupational Therapy, 71(2), 52-58. real world (Durham 1992, Roughton 2004, Watson 2004). Traditionally, the predischarge occupational therapy home assessment process involves taking older adults to their home for a short period of time and assessing their ability to perform some of the occupations of daily living within their own environment. Predischarge occupational therapy home assessments are routinely performed with older adults in Europe (Nygård et al 2000, Mountain and Pighills 2003), Australia (McEneany et al 2002, Mitchell and Unsworth 2004) and North America (Lysack and Neufeld 2003, Neufeld and Lysack 2004).

In the United Kingdom, there are often differences in health care trusts about the number of individuals involved in the planning of the home assessment and follow-up activities (McDonald et al 2003). Patterson et al (2001) suggested that senior therapists spend the equivalent of one day per week or more performing home assessments, with most NHS trusts performing between 11 and 40 assessments per month. Carers and other health and social care professionals involved in older adults' care and rehabilitation may also be present on the assessment.

Predischarge home assessments are complicated by the fact that an individual therapist's decision as to whether a predischarge home assessment is needed is based on his or her individual level of knowledge, clinical reasoning and experience (Welch and Lowes 2005). Moreover, the 
timing has to be right because this can have an impact upon the discharge decision-making process. There are no evidence-based protocols and so therapists usually assess the patient's functional ability using a non-structured observational checklist. However, therapists can apply standardised assessments within home assessments, for example the Westmead Home Safety Assessment (Clemson 1997). Clarke and Gladman (1995) have suggested that further investigation is needed to determine, for example, which patient group would benefit most from a home assessment. Consequently, it is hardly surprising that other health care professionals may challenge occupational therapists' assessment recommendations (Reich et al 1998).

It has been suggested that, in the acute care setting, the primary role of occupational therapists is to facilitate the discharge process and to undertake predischarge home assessments (McEneany et al 2002). In this environment, occupational therapists have expressed reservations about their assigned role because it is perceived to be limited to discharge facilitation or safe discharge (College of Occupational Therapists 2002). Moreover, it has been proposed that home assessments do not promote independence, but are derived from a compensatory approach that aims to resolve or compensate for functional deficits on discharge through the provision of assistive technology (Clark and Dyer 1998, Mountain and Pighills 2003). Consequently, occupational therapists have been challenged to consider whether home assessments focus upon short-term rather than long-term difficulties (Bore 1994). Indeed, Griffin (1993) has argued that economic constraints imposed on the health care system have resulted in occupational therapists adopting an assess/discharge approach to intervention as opposed to an assess/treat/discharge approach.

There is limited quality research demonstrating that these predischarge assessments enhance the health and wellbeing of older adults in acute care environments. Welch and Lowes (2005) attempted to discuss the evidence for home assessments within the discharge process and included fall prevention home visits as well as postdischarge visits. However, the review is very broad and, although acknowledging that more research needs to be undertaken and ignoring the quality of the research that has already been conducted in this area, the authors suggest that there is a 'body of evidence to support this intervention' (Welch and Lowes 2005, p158).

Surprisingly, very little research has explored predischarge home assessments from the occupational therapist's perspective. A search of several databases Allied and Complementary Medicine, 1985 to April 2006; British Nursing Index, 1994 to April 2006; Cinahl, 1982 to April 2006; Embase, 1974 to April 2006; King's Fund, 1979 to date; Medline, 1950 to April 2006; and the Cochrane Library - found only one published study.

A study by Nygård et al (2000) used a mixed method approach, including interviewing 23 older adults and a group interview with nine occupational therapists. Their findings suggest that the therapists strongly believed that these assessments were worthwhile, especially for the client's safety. The therapists judged that time was needed at home after discharge for older adults to discover current needs. However, the methodological reporting of the study is weak, since the study fails to give adequate information about the group interview with the occupational therapists and how the home visits were observed and recorded. Indeed, the authors admit that their study has numerous flaws and should be regarded as a reflective follow-up.

The rationale for carrying out the present study arose from clinical practice and discussions with numerous occupational therapists and managers who expressed concerns about predischarge home assessments in acute care. It was hoped that by ascertaining occupational therapists' perceptions of the home assessment, it would be possible to determine whether changes need to be made to the occupational therapy service or whether further research should be conducted to evaluate the effectiveness of predischarge home assessments. The aim of the study was to explore occupational therapists' perceptions of the value of predischarge home assessments.

\section{Method}

This article presents data from one part of a larger study, which also considers older adults' and carers' perspectives of predischarge home assessments; these are reported elsewhere (Atwal et al 2008). The research occurred on acute older adult wards, over 3 months, in a west London NHS trust. All occupational therapists who undertook predischarge home assessments in acute care with older adults during the duration of the study period were invited to participate and to provide a reflective log once a home assessment had been completed. The average age of the older adults who were taken on a home visit was 86.5 years. Nine of these older adults were female (Table 1).

Table 1. Patient characteristics

\begin{tabular}{|c|c|c|}
\hline Patient & Age (years) & Gender \\
\hline$P 1 \ldots$. & .........8. $84 \ldots$ & ...Female \\
\hline P2 ...... & ....95 _............... & ...Female \\
\hline P3...... & $\ldots . .88 \ldots \ldots$ & ....Male ... \\
\hline P4 ...... & .....91 ............. & ...Female \\
\hline P5 ..... & $\ldots .95 \ldots \ldots$ & ...Female \\
\hline P6...... & $\ldots . .84 \ldots \ldots$ & ...Female \\
\hline P7 .... & $\ldots . .84 \ldots \ldots \ldots$ & ...Female \\
\hline P8.... & $\ldots . .89$ & ....Male ... \\
\hline P9...... & $\ldots .73 \ldots \ldots$ & ...Male ... \\
\hline P10....... & .......92 _......... & ...Male ... \\
\hline P11...... & …...97 …...... & ...Female \\
\hline $\mathrm{P} 12 \ldots$. & $\ldots . . .81 \ldots \ldots$ & ...Female \\
\hline P13....... & $\ldots . . .76$ & ...Male ... \\
\hline P14....... & ….............. & ...Female \\
\hline P15....... & ….....86 _..... & ...Male ... \\
\hline
\end{tabular}


All seven eligible occupational therapists agreed to participate, and carried out 18 home assessments during this time frame; however, three reflective logs were not submitted because one therapist who had completed three home assessments subsequently left the trust. In total, 15 reflective diaries were completed by six therapists. One therapist conducted eight home assessments, one therapist conducted three home assessments and the remaining four therapists completed one assessment each.

In this study, a reflective diary was used as a self-evaluative account in which the occupational therapists recorded their own personal experiences, thoughts, feelings, impressions and observations, with a view to trying to understand their actions. A reflective diary was used rather than a focus group because the researchers wanted each therapist to reflect critically on his or her individual practice. Although focus groups can be regarded as a quick and convenient way to collect data, they use group interaction to explore perceptions and experiences (Kitzinger 1995). As the aim of the study was to capture individual perceptions, it was considered that the therapists would not feel comfortable about discussing individual cases and their own feelings. They might feel that they were being judged and confidentiality could be compromised.

The occupational therapists were asked to complete a reflective diary after each home assessment and were given an information sheet about the use of reflective diaries in practice and structured prompts to guide their reflection. In order to ensure confidentiality and for the therapists to be honest and objective about their perceptions and impressions, it was emphasised that only the research team would have access to the diaries. In addition, a therapist could ask for his or her reflective diary to be returned at any time and all the diaries were returned at the end of the research project after the data had been analysed. The participants were asked to complete the diaries as soon as possible after the home assessment when they perceived that they were ready to reflect upon the process.

Many professionals have incorporated reflection into practice as one means of ensuring best practice and personal development. Occupational therapists have been required to apply reflective thinking throughout their pre-registration education. Reflective diaries are useful for anyone who is undertaking a systematic review at his or her own practice and can also enhance critical self-reflection and self-awareness (Patton et al 1997, Biggs 1999).

The reflective diaries were based on answers to the following questions:

- How did you organise the home assessment? Was this a difficult process? Did you feel that the home assessment was necessary?

- How did you feel before the home assessment?

- What did you expect the home assessment/client to be like?

- To what extent were your expectations confirmed?

- What skills/methods did you use during the home assessment? What were you good at? What were you not so good at, and why?
- If you had a really good or a really bad home assessment, what did you learn from the experience?

- How did you feel at the end? Happy or sad, and why?

- Was the outcome satisfactory for you as the therapist and for the client?

- What were your perceptions about the older adults' quality of life?

- What will you do differently next week as a result of your reflections this week?

\section{Data analysis}

Thematic analysis is a method for searching, analysing and reporting themes within data (Braun and Clarke 2006, Fereday and Muir-Cochrane 2006). The phases of thematic analysis began with the first researcher (AA) reading the reflective diaries. The second step was to conceptualise the data by breaking down each sentence into something that represented an incident (Strauss and Corbin 1990). The interview was then coded, identifying both latent and manifest themes (Miles and Huberman 1994). Latent analysis deals with the underlying or implicit meaning of the text, which requires judgement and often depends on a coder's prior level of knowledge or expectation. Manifest analysis is the visible, surface content of a communication, such as how often a word appears or how often a word is mentioned (Boughner 2007, Ford 2007). These codes were as follows: preparation and planning; communication; expectations; anxiety and stressful; decision making; and feelings. Once particular phenomena in the data were identified, their labels were grouped together, which is referred to as 'categorising'. The categories that emerged were each given a name, and those that were related to one another were merged. Five themes were then created to link the underlying meaning that occurred within or across the categories (Graneheim and Lundman 2003):

- Theme 1: Home assessment process (process and preparation and planning)

- Theme 2: Benefits of the home assessment process (communication)

- Theme 3: Occupational therapists' expectations (expectations)

- Theme 4: Occupational therapists' anxiety (anxiety and stressful)

- Theme 5: Satisfaction and dissatisfaction with the outcome of the home visit assessment (decision making, feelings).

\section{Ethical considerations}

Ethical approval for the study was obtained initially from the university and then from a Local Research Ethics Committee (LREC) in February 2005, following NHS REC application procedures. The research team was independent of the occupational therapy team. The participants were informed that the reflective diaries were confidential and that the information provided would subsequently be anonymous, would be stored securely and would be destroyed 2 years after the study was completed. 


\section{Findings}

\section{Home assessment process}

All the home assessments were planned by an occupational therapist, and were arranged by liaising with patients' relatives and carers. All the occupational therapists perceived that visits were relatively easy to organise, with most therapists focusing upon how access could be gained to the patient's home. One therapist did arrange for clothes to be brought into hospital. However, one therapist perceived that more time was needed to plan a home assessment, '... it was last minute', whereas another therapist felt that more time could be spent in explaining to the older adult the purpose of the home assessment. One therapist considered that forward planning was an important aspect of a successful home assessment. Other therapists identified the rationale for carrying out home assessments to be because of family concerns or a lack of acknowledgement by the patient of the potential difficulties:

I did not feel that a visit was necessary however after speaking with the daughter I realised the patient would have to manage at home with limited assistance.

\section{Benefits of the home assessment}

The most cited reasons for carrying out home assessments were the patient's mobility problems, assessing the suitability of the home environment, and changes to the patient's functional status. One occupational therapist wrote: 'I could see a home visit was warranted due to changes in functional ability.' Another stated: 'I did feel that the home visit was necessary to ensure the home environment was set up for the patient's needs ... and to ensure patients can manage mobility in the home.'

Some occupational therapists highlighted the importance of listening to the family's concerns and how this had had an impact upon the discharge process:

I felt that I was a very good listener on the assessment.

Many of the family's concerns did not require any action they really just wanted to be heard.

In addition, the home assessment had been a catalyst to discuss the future placement needs:

I think I was good at making the patient understand the realities of coming home and the care/assistance/modifications that would be required. The patient lacked insight into issues and seemed to think that when she came home she would be like she was before.

Another occupational therapist regarded the home assessment as an important aspect in the rehabilitation process, stating: 'I was actually looking forward to the assessment as it was a big step to get this patient to this stage.' One therapist felt that one patient was able to accept changes in her occupational routine as she had performed these daily occupations on the home assessment: '... Patient found it difficult to accept changes ... Insight was increased, whilst letting patient take part in tasks with supervision.'

\section{Occupational therapists' expectations}

The patient's functional ability and the patient's own concerns did have an impact upon the occupational therapists' expectations of the home assessment process. One therapist reported that the home assessment had confirmed an expectation that there would be an issue with the patient's functional mobility:

I expected that the home assessment would be quite difficult. The patient is concerned about returning home due to ... significant decline in physical function.

I expected the patient to need frequent rests, expected the client to show her ability, expected to find bed transfers difficult, find a supportive network within warden controlled flat, expected the patient would require support on discharge.

By completing a home assessment, the occupational therapist was able to find out how the patient was specifically mobilising within the home environment and discovered that perceived expectations were confirmed:

Patient was mobilising with a frame, but also had rails in place prior to having a walking aid. Therefore, patient was used to walking around the house using the grab rails for support and not being provided with a walking frame was confused as to whether she was walking with the walking aid or rails and as a result was at increased risks of falls.

In contrast, the same therapist reflecting on another patient stated that expectations about a patient's level of functioning within the home environment had not been proven to be correct:

I expected the patient's level of function would be much improved in the home, as these would be familiar surroundings. This was also what OTs had found on a previous home assessment ... My expectations were not confirmed to any extent. The patient's level of function did not improve to any extent on the assessment; she remained confused and disorientated even within her own environment.

\section{Occupational therapists' anxiety}

In the reflective diaries, anxiety was expressed about aspects of the home assessment process. Some of these were related to an older adult's level of functioning or ill health, for example:

I was slightly anxious about how the patient might cope on the assessment as she had decreased mobility and increased shortness of breath ... It was decided that we would take an oxygen cylinder as a precaution. This also made me less anxious.

Before the home assessment I was quite anxious about how this assessment would go as the patient at times became very anxious when mobilising and would not put weight on her foot. I was anxious that she might do this on the assessment. I was also anxious that the patient may fall on the assessment, as she is very unsteady when mobilising and requires close supervision. 
Another occupational therapist stated that she felt confident at the end of the visit as the patient did not have any cognitive difficulties:

I felt very confident that despite the patient's decreased functional level this would be a successful discharge. The patient was very rational and logical and has good safety awareness, which puts me at ease when planning discharges.

The occupational therapists perceived that home assessments could be personally tiring owing to the type and amount of communication that occurred with older adults and their carers:

I felt very tired as I had to do lots of talking/explaining etc and I had to encourage the patient constantly with her mobility.

An occupational therapist also acknowledged a patient's anxiety on the home assessment:

The patient was very anxious on the assessment and the assessment was lengthy due to the number of issues that were needed to be talked through/problem solved. The patient mobility/physical function did not improve on the assessment ... she required close supervision to prevent falls from occurring.

Similarly, another therapist emphasised the importance of remembering that a home assessment can be a stressful process.

\section{Satisfaction and dissatisfaction with the outcome of the home assessment}

In most of the reflective diaries, the occupational therapists were happy or satisfied with the outcome of the home assessments because in most cases they perceived that the aims of the assessment had been met and/or that the carer had felt the assessments to be useful. In two instances, one therapist stated that she felt both 'happy and relieved'. One therapist felt that the home assessment had helped to diminish anxiety about the transition from hospital to home:

Although the assessment did not highlight any major issues, in hindsight an access assessment probably would have been adequate. I still feel that it was a good opportunity to assist the patient and family in the transition from hospital to home ... The family were quite anxious about the discharge.

Another therapist was of a similar opinion:

I learnt that although the assessment was lengthy and we spent a lot of time talking through problems associated with going home, this in the end was beneficial time spent, as the patient felt more confident at the conclusion of the assessment that going home was a possibility.

One therapist felt that the outcome was not satisfactory because the patient was not able to return home. Another therapist recorded an instance when a carer was limiting an older adult's occupation, which resulted in the therapist reflecting about how therapists managed differences of opinion:
I have concerns about this lady's quality of life. The daughter insisted that if her mother was to return home, then she was not to use the stair lift and that she was to stay upstairs. This means that the patient is confined to the bedroom and the sitting room. I felt that the patient was safe to access all areas of the house and could have been independent in doing so, for example being able to make a cup of tea. I feel that this would have improved her quality of life ... I was somewhat frustrated however, that the patient was going to be limited in accessing her home due to the daughter's stipulations.

The same therapist wondered if an older adult's carer should have been challenged in order to achieve the best outcome for the patient:

I am unsure due to the significant family input that plans were made according to what the patient wanted, as opposed to what the family wanted ... I felt I could have challenged the daughter more about her reasons for deciding that her mother had to stay upstairs.

Another therapist reported that an attempt had been made to address concerns with the patient; however, this was difficult due to the patient's cognitive impairment:

I attempted to explain to the patient why we had concerns about her returning to independent living. I felt I was good at prompting at the issues and highlighting the concerns, however due to the patient's cognitive impairment, she was not able to gain insight into her current level of function.

\section{Discussion}

For occupational therapists, the home assessment enabled them primarily to assess an older adult's functional ability within the home environment. Indeed, the most cited reasons for carrying out home assessments were mobility concerns and to assess the suitability of the home environment. Similar findings have been reported by Watson (2004), who conducted a Delphi study with occupational therapists working in orthopaedics, and by Roughton (2004), who conducted a study with occupational therapists working with older adults. Although both these unpublished studies had a poor response rate, they did reach similar conclusions: the most important aim of a home visit was to assess a patient's ability to cope at home in order to facilitate discharge planning, and the second most important aim was to assess a patient's functional capabilities within his or her own home environment. Interestingly, with regard to assessing the environment, this was cited as the third most important aim of the assessment by Watson (2004) and the fifth most important aim of the assessment by Roughton (2004).

Some researchers have identified the benefits of the home visit assessment. Marshall (2004) explored four occupational therapists' perceptions of home assessments 
in an intermediate care setting. Although the study remains unpublished, it offers some useful insights into occupational therapists. This study found that occupational therapists believed that home assessments were a means to provide both the patient and the therapist with an understanding of the patient's strengths and weaknesses; that home assessments informed aims and objectives; and that home assessments offered the most appropriate place in which to assess risk factors in the home. The home assessment was perceived as being important because it allowed the therapist to meet and communicate with the patient's social network. In contrast in the present study, the predischarge home assessments seemed to focus on mobility, transfers and risk elimination. Interestingly, the therapists did not find this practice restrictive. Occupational therapists in acute care environments need to ensure that visits meet the aims of older adults and enable them to discuss their fears, hopes and occupational goals. This may have resource implications for trusts; however, it could greatly enhance the discharge experience for older adults.

This study found that occupational therapists, as opposed to patients, often initiated home assessments. It is interesting that the therapists did not usually cite carers' concerns as the prime reason for carrying out the home assessment, although there is evidence that the therapists did value the importance of predischarge home assessments for carers. McDonald et al (2003), who investigated current practice relating to predischarge home assessments with older adults, found that only $37.2 \%$ of the respondents $(62 / 166)$ said that patients were involved in identifying the need for a home assessment. Similar findings have been reported in an unpublished study by Moats (2004), who found that occupational therapists' interpretations of client-centred practice were somewhat limited and that, in the acute setting, older adults were often not included in decision making. The therapists found it difficult to manage the competing issues of safety and autonomy and often did not consider what occupations a patient would have when discharged to a new environment. Similarly, Nygård et al (2000) found that occupational therapists perceived that more time was needed at home after discharge for the older adult to discover current needs.

Occupational therapists need to ensure that patients understand the aims of the home visit and have been given adequate warning and information (Atwal et al 2008). Departments need to consider the best way to present both written and verbal information to older adults. Moreover, perhaps older adults should sign a consent form outlining that they understand the aims of the visit. More research is needed into the long-term benefits of home visits and the value of postdischarge home visits.

In the literature, there has been some reference to patient anxiety within the context of home assessments (Bore 1994, Clark and Dyer 1998, Davies 2004), although there is evidence that older adults do find the home assessment helpful (Nygård et al 2000, Hibberd 2004).
The occupational therapists in the present study were also aware that the home assessment could be anxiety provoking and this research supports the evidence in the grey literature (Marshall 2004). Previous research has found that home assessments were enjoyed by therapists (Marshall 2004, Mitchell and Unsworth 2004, Moats 2004). Although the findings from the present research suggest that the occupational therapists also experienced feelings of anxiety, their anxiety was related primarily to the older adults' level of functioning or ill health. In most cases, the occupational therapists were happy with the assessment if the outcomes were positive. In order to minimise occupational therapy anxiety about the home visit process, it is essential that there is clinical reasoning of the positive and negative aspects of taking older adults on home visits. Occupational therapists could consider whether the patient has reached his or her maximum level of functioning or consider other intermediate services to enable older adults to regain lost skills.

\section{Methodological considerations}

This qualitative study explored the perceptions of occupational therapists in relation to the home assessment process in one NHS trust only, and therefore the findings cannot be generalised to all other acute care settings. Only six therapists were involved in keeping reflective diaries and their individual skills and expertise in the field of acute care occupational therapy could have had an impact on their perception of home assessments. One therapist completed 8 out of the 15 diaries, which could have biased the findings.

\section{Conclusion}

The findings from this study suggest that the predischarge home assessments seemed to focus on mobility, transfers and risk elimination. The occupational therapists' satisfaction and dissatisfaction with the outcome of the home assessment were related to the incidents that occurred on the assessment. Some of the occupational therapists' anxieties were related to the older adults' level of functioning or ill health.

Occupational therapists need to ensure that older adults are involved in the predischarge decision-making process and that they take into account the occupational goals of each older adult. Therapists need to be able to reason clinically the rationale for the home visit and how it could enhance an older adult's quality of life. Strategies to facilitate this could involve all patients consenting to a visit; spending time discussing the recommendations following the visit; and, ultimately, the older adult signing a copy of the home visit report.

\section{Acknowledgements}

Thanks are extended to the West Middlesex Hospital Research and Development Department who funded this research, and to the occupational therapists who participated in the study. 


\section{References}

Atwal A, McIntyre A, Craik C, Hunt J (2008) Older adults and carers' perceptions of pre-discharge occupational therapy home visits in acute care: a qualitative study. Age and Ageing, 37(1), 72-76.

Biggs J (1999) Teaching for quality learning at university. Buckingham: Society for Research into Higher Education and Open University Press.

Bore J (1994) Occupational therapy home visits: a satisfactory service? British Journal of Occupational Therapy, 57(3), 85-88.

Boughner R (2007) Research methods. Nonreactive research: content analysis.

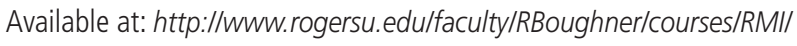
Content\%20analysis\%20outline. html Accessed on 07.01.08.

Braun V, Clarke B (2006) Using thematic analysis in psychology. Qualitative Research in Psychology, 3, 77-101. Available at: wwwQualResearchPsych.com Accessed on 10.04.07.

Clark H, Dyer S (1998) Equipped for going home from hospital. Health Care in Later Life, 3(1), 35-45.

Clarke PA, Gladman JR (1995) A survey of pre-discharge occupational therapy home visits for stroke patients. Clinical Rehabilitation, 9(4), 339-42.

Clemson L (1997) Home fall hazards: a guide to identifying fall hazards in the homes of elderly people and an accompaniment to the assessment tool, the Westmead Home Safety Assessment (WeHSA). West Brunswick, Victoria: Co-ordinates Publications.

College of Occupational Therapists (2002) From interface to integration: a consultation document. London: COT.

Davies S (2004) An investigation into the experience of occupational therapy home visits for carers of older people. Unpublished MSc thesis. Eastbourne: University of Brighton.

Department of Health (2000) The NHS Plan: a plan for investment, a plan for reform. London: Stationery Office.

Durham D (1992) Occupational and physical therapists' perspective of the perceived benefits of a therapeutic home visit program. Physical and Occupational Therapy in Geriatrics, 10(3), 15-33.

Fereday J, Muir-Cochrane E (2006) Demonstrating rigor using thematic analysis: a hybrid approach of inductive and deductive coding and theme development. International Journal of Qualitative Methods, 5(1), Article 7. Available at: http://www.ualberta.ca/ iiqm/backissues/ 5_1/html/fereday.htm Accessed on 08.04.07.

Ford M (2007) Graduate research methods. Available at: $h t t p: / / p e g a s u s . c c$. ucf.edu/ cjreg/Grmlecture3.htm Accessed on 07.01.08.

Graneheim UH, Lundman B (2003) Qualitative content analysis in nursing research: concepts, procedures and measures to achieve trustworthiness. Nurse Education Today, 24(2), 105-12.

Griffin SD (1993) Short bed stays: their effect on occupational therapy services in teaching hospitals. Archives of Physical Medicine and Rehabilitation, 74(10), 1087-90.

Hibberd J (2004) An evaluation of the home visiting process within the in-patient intermediate care services. Unpublished report. Norwich: School of Allied Health Professions, University of East Anglia.

Kitzinger J (1995) Qualitative research: introducing focus groups. British Medical Journal, 311(7000), 299-302.
Lysack CL, Neufeld S (2003) Occupational therapists' home evaluations: inequalities, but doing the best we can. American Journal of Occupational Therapy, 57(4), 369-79.

Marshall KJ (2004) How do occupational therapists experience pre-discharge home visits? Unpublished MSc thesis. Eastbourne: University of Brighton.

McDonald RE, Chesson RA, Burnett G, Funai GJ, Holdsworth LK, Upton CDJ (2003) Pre-discharge home assessment visits with elderly patients. Collaborative Study of Current Practice. Physiotherapy, 89(5), 323.

McEneany J, McKenna K, Summerville P (2002) Australian occupational therapists working in adult physical dysfunction settings: what treatment media do they use? Australian Occupational Therapy Journal, 49(3), 115-27.

Miles MB, Huberman AM (1994) Qualitative data analysis. 2nd ed. Thousand Oaks, CA: Sage.

Mitchell R, Unsworth CA (2004) Role perceptions and clinical reasoning of community health occupational therapists undertaking home visits. Australian Occupational Therapy Journal, 51(1), 13-27.

Moats G (2004) Discharge accommodation decision making with older people: the experience of occupational therapists. Unpublished MSc thesis. Halifax, Nova Scotia: Dalhousie University.

Mountain G, Pighills A (2003) Pre-discharge home visits with older people: time to review practice. Health and Social Care in the Community, 11(2), 146-54.

Neufeld S, Lysack C (2004) Allocation of rehabilitation services: who gets a home evaluation? American Journal of Occupational Therapy, 58(6), 630-38.

Nygård N, Grahn U, Rudenhammer A, Hydling S (2000) Reflecting on practice: are home visits prior to discharge worthwhile in geriatric inpatient care? Scandinavian Journal of Caring Sciences, 18(2), 183-203.

Patterson CJ, Viner J, Saville C, Mulley GP (2001) Too many pre-discharge home visits for older patients? A postal questionnaire survey. Clinical Rehabilitation, 15(3), 291-95.

Patton JG, Woods SJ, Agarenzo T (1997) Enhancing the clinical practice experience through journal writing. Journal of Nursing Education, 36(5), 238-40.

Reich S, Eastwood C, Tilling K, Hopper A (1998) Clinical decision making, risk and occupational therapy. Health and Social Care in the Community, 6(1), 47-54.

Roughton F (2004) Why do occupational therapists carry out pre-discharge home visits with hospital in-patients? An investigation using the Delphi technique. Unpublished MSc thesis. Eastbourne: University of Brighton.

Strauss A, Corbin J (1990) Basics of qualitative research. Newbury Park, CA: Sage.

Watson L (2004) Why do occupational therapists carry out pre-discharge home visits with hospital in-patients? Unpublished MSc thesis. Eastbourne: University of Brighton.

Welch A, Lowes S (2005) Home assessment visits within the acute setting: a discussion and literature review. British Journal of Occupational Therapy, 68(4), 158-64. 